\title{
Population structure and geographical segregation of Cryptosporidium parvum Ild subtypes in cattle in China
}

\author{
Zhenjie Zhang ${ }^{1,2+}$, Suhui Hu ${ }^{1 \dagger}$, Wentao Zhao ${ }^{1}$, Yaqiong Guo ${ }^{1}, \mathrm{Na} \mathrm{Li}^{1}$, Zezhong Zheng ${ }^{1}$, Longxian Zhang ${ }^{3}$, \\ Martin Kváč ${ }^{4}$, Lihua Xiao ${ }^{1,2^{*}}$ (10 and Yaoyu Feng ${ }^{1,2^{*}}$
}

\begin{abstract}
Background: Cryptosporidium parvum is a zoonotic pathogen worldwide. Extensive genetic diversity and complex population structures exist in C. parvum in different geographical regions and hosts. Unlike the lla subtype family, which is responsible for most zoonotic C. parvum infections in industrialized countries, Ild is identified as the dominant subtype family in farm animals, rodents and humans in China. Thus far, the population genetic characteristics of Ild subtypes in calves in China are not clear.
\end{abstract}

Methods: In the present study, 46 C. parvum isolates from dairy and beef cattle in six provinces and regions in China were characterized using sequence analysis of eight genetic loci, including msc6-7, rpgr, msc6-5, dz-hrgp, chom3t, hsp70, mucin1 and gp60. They belonged to three Ild subtypes in the gp60 gene, including IIdA20G1 ( $n=17)$, IIA19G1 $(n=24)$ and IIdA15G1 $(n=5)$. The data generated were analyzed for population genetic structures of $C$. parvum using DnaSP and LIAN and subpopulation structures using STRUCTURE, RAXML, Arlequin, GENALEX and Network.

Results: Seventeen multilocus genotypes were identified. The results of linkage disequilibrium analysis indicated the presence of an epidemic genetic structure in the C. parvum Ild population. When isolates of various geographical areas were treated as individual subpopulations, maximum likelihood inference of phylogeny, pairwise genetic distance analysis, substructure analysis, principal components analysis and network analysis all provided evidence for geographical segregation of subpopulations in Heilongjiang, Hebei and Xinjiang. In contrast, isolates from Guangdong, Shanghai and Jiangsu were genetically similar to each other.

Conclusions: Data from the multilocus analysis have revealed a much higher genetic diversity of $C$. parvum than gp60 sequence analysis. Despite an epidemic population structure, there is an apparent geographical segregation in C. parvum subpopulations within China.

Keywords: Cryptosporidium parvum, Epidemic, Geographical segregation, Cattle, China

*Correspondence: Ixiao1961@gmail.com; yyfeng@scau.edu.cn ${ }^{\dagger}$ Zhenjie Zhang and Suhui Hu: Equal contributors

${ }^{1}$ Center for Emerging and Zoonotic Diseases, College of Veterinary

Medicine, South China Agricultural University, Guangzhou, Guangdong 510642, China

Full list of author information is available at the end of the article

\section{Background}

Cryptosporidium spp. are apicomplexan pathogens that can cause debilitating gastrointestinal illness in animals and humans with the main clinical symptom as diarrhea [1]. There is extensive genetic variation within the genus Cryptosporidium. Among the nearly 40 Cryptosporidium species identified, C. parvum is the most important species causing zoonotic cryptosporidiosis [2]. It has a wide host range, with over 20 subtype families based on

(c) The Author(s) 2020. This article is licensed under a Creative Commons Attribution 4.0 International License, which permits use, sharing, adaptation, distribution and reproduction in any medium or format, as long as you give appropriate credit to the original author(s) and the source, provide a link to the Creative Commons licence, and indicate if changes were made. The images or other third party material in this article are included in the article's Creative Commons licence, unless indicated otherwise in a credit line to the material. If material is not included in the article's Creative Commons licence and your intended use is not permitted by statutory regulation or exceeds the permitted use, you will need to obtain permission directly from the copyright holder. To view a copy of this licence, visit http://creativeco mmons.org/licenses/by/4.0/. The Creative Commons Public Domain Dedication waiver (http://creativecommons.org/publicdomain/ zero/1.0/) applies to the data made available in this article, unless otherwise stated in a credit line to the data. 
sequence analysis of the $60 \mathrm{kDa}$ glycoprotein (gp60) locus [3]. Among the most common subtype families, IIa and IId are zoonotic, while IIc and IIe are anthroponotic $[2,4]$.

Cattle are among the most common hosts of C. par$v u m$, with pre-weaned calves being considered the most important reservoir for zoonotic C. parvum infection [5]. Differences in virulence and transmission dynamics of $C$. parvum have been observed among geographical regions [6]. Subtyping of C. parvum in bovine studies identified an exclusive occurrence of IId subtypes in calves in China, mostly IIdA15G1 and IIdA19G1 [7]. Moreover, these IId subtypes have caused outbreaks of cryptosporidiosis in calves in several areas in China, leading to the occurrence of significant mortality $[8,9]$. In contrast, pre-weaned calves in industrialized countries are mostly infected with C. parvum IIa subtypes, especially IIaA15G2R1 [6, 7].

Population genetic studies based on highly polymorphic loci can shed light on the true genetic diversity of $C$. parvum in disease endemic areas and compensate for the relatively low resolution of the single $g p 60$ locus because of the likely occurrence of genetic recombination among loci and the existence of genetic determinants of other phenotypic traits $[3,10]$. Multilocus typing tools based on genetic loci with simple tandem repeats have been used in studies of the population genetic characteristics of $C$. parvum, leading to the discovery of high genetic diversity, significant geographical segregation and complex population structure $[11,12]$. Thus far, a range of genetic structures of C. parvum have been identified, including panmictic (unrestricted gene flow and linkage equilibrium among loci), clonal (largely restricted gene flow and linkage disequilibrium among loci), and epidemic (underlying panmictic structure masked by an abundance of genetically identical clones) [2].

Most previous studies of the population genetics of C. parvum had focused on the IIa subtype family. A mostly panmictic population structure for C. parvum IIa subtype family has been found in humans and calves in many industrialized nations [12-21]. This could be related to the transmission intensity and reproductive characteristics of the IIa subtype family. Indeed, IIa subtypes, especially the hyper-transmissible IIaA15G2R1, are the dominant ones in cattle and humans in these countries [2]. In addition, one study of IIaA15G2R1 has also shown an epidemic population structure and common occurrence of genetic recombination within the subtype [16]. Several analyses of the IId subtype family have demonstrated potential differences in population structure between IId and IIa subtype families. For example, IIa subtypes in cattle in Spain has a panmictic structure while IId subtypes in sheep has a clonal structure [20,22]. This was supported by a population genetic study of the
C. parvum IId subtype family in China, Egypt and Sweden, which mostly has a clonal population structure.

The aim of this study was to explore the population genetic characteristics of IId subtypes of C. parvum in cattle in China using multilocus sequence typing (MLST) of isolates.

\section{Methods}

\section{Sample sources}

Forty-six isolates of $C$. parvum IId subtypes including IIdA20G1 $(n=17)$, IIdA19G1 $(n=24)$, IIdA15G1 $(n=$ 5) from beef and dairy cattle in Xinjiang, Heilongjiang, Hebei, Shanghai, Jiangsu and Guangzhou, China, were selected for the population genetics analysis. They were from previous and ongoing studies of molecular epidemiology of cryptosporidiosis in cattle [8, 23, 24]. The geographical distribution of isolates and their gp 60 subtype designations are shown in Table 1 and Fig. 1 . The six provinces and autonomous regions are representative ones in China, including the south (Guangdong), east (Shanghai and Jiangsu), center (Hebei), northeast (Heilongjiang) and northwest (Xinjiang). These areas have some of the largest dairy farms in China. The three C. parvum subtypes examined in the study are the most common subtypes in China, responsible for over $90 \%$ C. parvum infections in cattle. They were diagnosed by DNA sequence analysis of the gp60 gene [23].

\section{PCR and sequence analyses}

Eight polymorphic loci including gp60 with simple tandem repeats were used in the characterization of C. par$v u m$ isolates in the present study. In addition to gp60, they included $m s c 6-7$ (serine repeat antigen), rpgr (retinitis pigmentosa GTPase regulator), msc6-5 (hypothetical trans-membrane protein), $d z$-hrgp (hydroxyproline-rich glycoprotein), chom $3 t$ (T-rich gene fragment), hsp70 (70 $\mathrm{kDa}$ heat shock protein), mucin1 (mucin-like protein). Nested PCR was used in the analysis of these genetic loci as previously described [25]. Each isolate was analyzed twice by PCR at each genetic locus. Reagent-grade water was used as a negative control, whereas DNA of C. parvum IOWA isolate (IIaA15G2R1 subtype) was used as a positive control. Positive PCR products were sequenced on an ABI 3730 Genetic Analyzer (Applied Biosystems, CA, USA). The sequences generated were assembled using ChromasPro v.2.1.8 (http://technelysium.com.au/ ChromasPro.html) and aligned with reference sequences from each locus using the program Clustal X v.2.1 (http:// www.clustal.org/).

\section{Population genetic analyses}

The sequences from the eight loci were tandemly concatenated for each isolate. The multilocus genotypes 
Table 1 Subtypes and regional origins of Cryptosporidium parvum isolates used in the present study

\begin{tabular}{|c|c|c|c|c|c|}
\hline Location & Farm & Host & Subtypes & No. of isolates & Reference \\
\hline \multirow[t]{2}{*}{ Guangdong } & $\mathrm{D}$ & Dairy cattle & IIAA19G1 & 5 & {$[23]$} \\
\hline & $\mathrm{E}$ & Dairy cattle & IdA19G1 & 5 & Present study \\
\hline \multirow[t]{2}{*}{ Hebei } & G & Dairy cattle & $\| d A 20 G 1$ & 5 & Present study \\
\hline & $\mathrm{H}$ & Beef cattle & $\| d A 20 G 1$ & 7 & Present study \\
\hline Heilongjiang & $F$ & Dairy cattle & $\| d A 20 G 1$ & 5 & Present study \\
\hline Jiangsu & A & Dairy cattle & IIA19G1 & 4 & {$[8]$} \\
\hline \multirow[t]{2}{*}{ Shanghai } & B & Dairy cattle & IIA19G1 & 5 & [24] \\
\hline & C & Dairy cattle & $\| d A 19 G 1$ & 5 & [24] \\
\hline Xinjiang & । & Dairy cattle & $\| d A 15 G 1$ & 5 & Present study \\
\hline Total & & Dairy cattle, Beef cattle & $\begin{array}{l}\text { IdA19G1, \|dA20G1, } \\
\text { IdA15G1 }\end{array}$ & 46 & \\
\hline
\end{tabular}

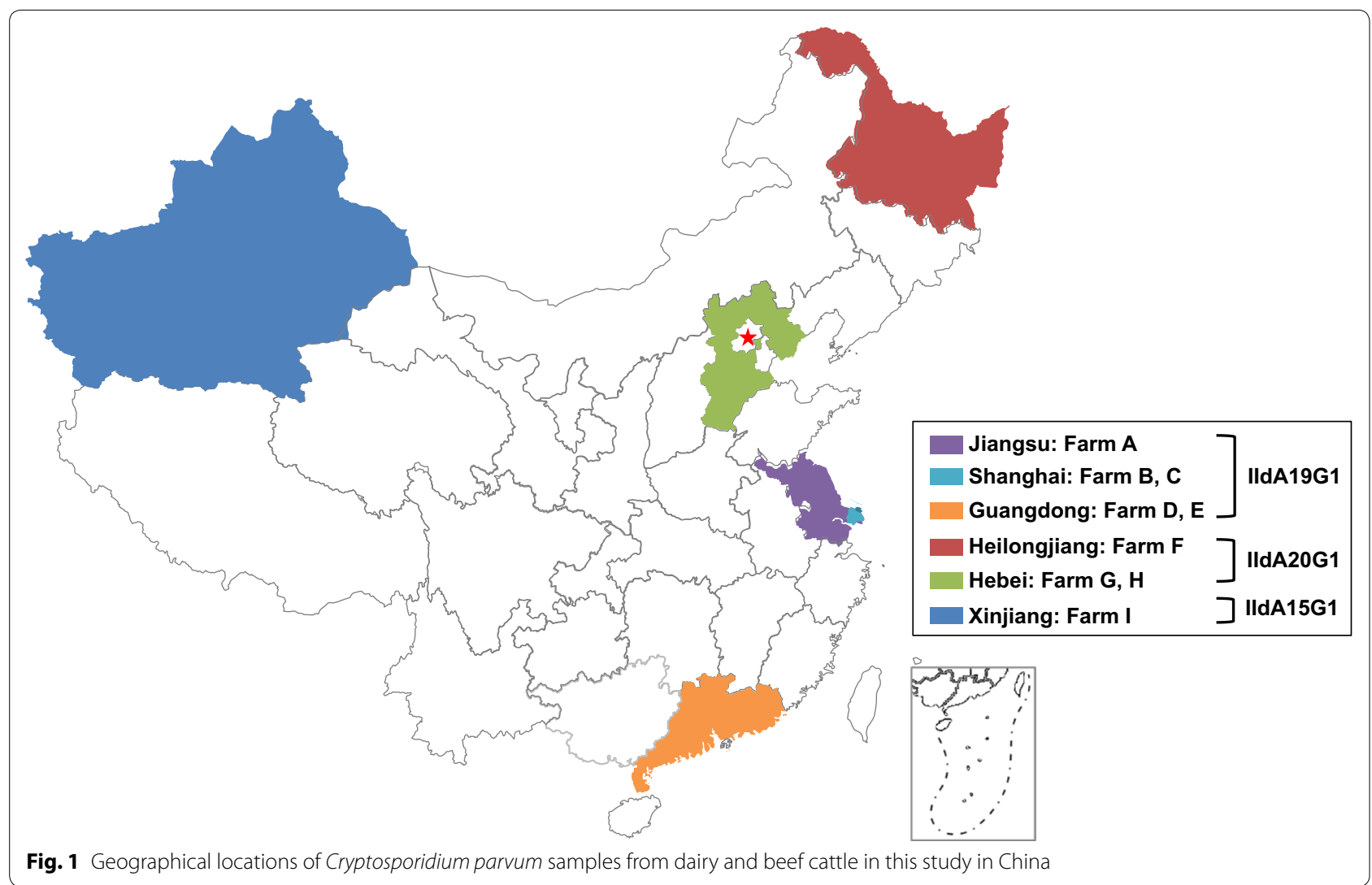

(MLGs) with the same sequences were analyzed for gene diversity $(H d)$, linkage disequilibrium $(L D)$ and recombination events (Rms) using software DnaSP version 6.12 .03 (http://www.ub.edu/dnasp/) with consideration of both sequence length polymorphism and nucleotide substitutions [26]. The genetic structure of C. parvum IId subtypes was assessed by measuring the association of standard correlation index $\left(I_{A}^{S}\right)$ and the relationship between $V_{D}$ and $L$ using the online software LInkage
ANalysis, v.3.7 (http://guanine.evolbio.mpg.de/cgi-bin/ lian/lian.cgi.pl/query) [27].

\section{Substructure analyses}

Maximum likelihood analysis implemented in the software RAxML v.8.0.0 (http://epa.h-its.org/raxml/submit single_gene) was used in clustering nucleotide sequences of all isolates using the General Time Reversible (GTR) model [28]. Subpopulations within the 46 isolates of the 
Table 2 Genetic diversity within Cryptosporidium parvum populations based on analysis of the concatenated sequences from eight genetic loci

\begin{tabular}{llllll}
\hline Population & $n$ & $H$ & $H d$ & $\operatorname{LD}\left(\left|D^{\prime}\right|\right)$ & $R m s$ \\
\hline Location & & & & & \\
Guangdong & 10 & 6 & 0.78 & $\mathrm{Y}=1.0000+0.0000 \mathrm{X}$ & 0 \\
Hebei & 10 & 1 & 0.00 & - & - \\
Heilongjiang & 5 & 1 & 0.00 & - & - \\
Jiangsu & 4 & 2 & 0.67 & - & 0 \\
Shanghai & 9 & 7 & 0.94 & $\mathrm{Y}=0.9295+0.0350 \mathrm{X}$ & 1 \\
Xinjiang & 3 & 3 & 1.00 & $\mathrm{Y}=1.0000+0.0000 \mathrm{X}$ & 0 \\
gp60 subtypes & & & & & \\
IIdA19G1 & 23 & 12 & 0.85 & $\mathrm{Y}=0.8811+0.0177 \mathrm{X}$ & 1 \\
IIdA20G1 & 15 & 2 & 0.48 & - & 0 \\
IIdA15G1 & 3 & 3 & 1.00 & $\mathrm{Y}=1.0000+0.0000 \mathrm{X}$ & 0 \\
Total & 41 & 17 & 0.89 & $\mathrm{Y}=0.9994-0.0041 \mathrm{X}$ & 1 \\
\hline
\end{tabular}

Abbreviations: $n$ number of multilocus genotypes, $H$ number of haplotypes (types based on SNPs alone), Hd gene diversity; LD (ID'I), linkage disequilibrium between sites, where $\mathrm{X}$ is the nucleotide distance (measured in kilobases); Rms minimum number of recombination events

C. parvum IId subtype family were identified using STRU CTURE v.2.3.4 (http://web.stanford.edu/group/pritc hardlab/structure.html) [29]. Several analyses of allelic data were performed by using $\mathrm{K}$ (likely populations) ranging from 2 to 10 and 50,000 iterations after a 'burnin' of 50,000 iterations. Output at $\mathrm{K}=3-5$ provided the best fit to MLST data and was used in further analyses. Pairwise genetic distance $\left(F_{\mathrm{st}}\right)$ was calculated using Arlequin v.3.5 (http://cmpg.unibe.ch/software/arlequin3/) in the evaluation of the genetic differentiation between MLGs of C. parvum. Principal coordinates analysis (PCoA) via covariance matrix with data standardization was performed on the generated matrices with the software GENALEX v.6.501 (http://biology-assets.anu.edu. au/GenAlEx) [30]. A median-joining phylogeny was generated using Network software v.5.0 (www.fluxus-engin eering.com/sharenet.htm) to estimate the genetic segregation and evolutionary trend of C. parvum [31].

\section{Results}

\section{MLST subtypes and sequence polymorphism}

Forty-one of the 46 isolates were successfully amplified at all eight loci. Among them, dz-hrgp, rpgr and mucin1 had relatively higher sequence polymorphism, with 5, 4 and 4 subtypes being identified, respectively. In contrast, the 44 isolates generated the same sequence at the normally polymorphic chom $3 t$ locus (Additional file 1: Table S1). Altogether, 17 MLGs were obtained from these isolates of C. parvum. Among them, the IIdA19G1 isolates from Guangdong, Jiangsu and Shanghai consisted of 12 MLGs. In addition, the IIdA20G1 isolates from Hebei and Heilongjiang had two geographically segregated MLGs. The IIdA15G1 isolates from Xinjiang had 3 different MLGs (Additional file 1: Table S1).

Sequence data of all eight loci were concatenated to make a multilocus contig of $4740 \mathrm{bp}$ in length. There was a high genetic diversity $(H d=0.89)$ within $C$. parvum IId population in China (Table 2). Among the IIdA19G1 isolates, the genetic diversity of isolates from Shanghai $(H d=0.94)$ was greater than isolates from Guangdong $(H d=0.78)$ or Jiangsu $(H d=0.67)$ (Table 2). This could be attributed to the difference in the number of farms examined in different regions. In contrast, IIdA20G1 isolates had relatively low genetic diversity ( $H d=0.48)$. Among them, isolates from Hebei and Heilongjiang showed high genetic homogeneity $(H d=0.00)$ within each population. In contrast, IIdA15G1 isolates from Xinjiang were highly heterogeneous $(H d=1.00)$ (Table 2$)$.

\section{Population structure of Ild subtypes of $C$. parvum}

In the analysis of the genetic structure of IId subtypes with $V_{D}$ and $L$ measurements, an epidemic genetic structure was obtained in the overall population $\left(I_{A}^{S}\right.$ $=-0.0421, P_{M C}=0.889$ and $\left.V_{D}: 1.1307<L: 2.3307\right)$ (Table 3). In further analyses, most of the subpopulations by region or gp60 subtype also had the epidemic genetic structure, except for the subpopulations of Heilongjiang, Hebei and Xinjiang which could not be determined due to the small sample size (Table 3 ).

\section{Subpopulations of Ild subtypes of $C$. parvum}

Maximum likelihood analysis of the sequences grouped the 41 isolates into several evolutionary clusters (Fig. 2). Among them, IIdA20G1 isolates from Heilongjiang formed one cluster separated from other isolates including IIdA20G1 isolates from Hebei. Another cluster was formed by IIdA15G1 isolates from Xinjiang. In contrast, there was no significant geographical clustering among IIdA19G1 isolates from Jiangsu, Shanghai and Guangdong (Fig. 2).

A similar result was obtained in STRUCTURE analysis of allelic data. At all K-values used in the analysis, the IIdA20G1 isolates from Heilongjiang were clearly separated from isolates of other regions, including those from Hebei that had the same gp60 subtype. The best separation of subpopulations by $g p 60$ subtype was seen at a K-value of 3 ; all three $C$. parvum subtypes formed their own clusters (Fig. 3). In addition, regardless the K-values (3-5) used in the analyses, IIdA19G1 isolates from Guangdong, Shanghai and Jiangsu clustered together (Fig. 3). This was supported by the results of PCoA and median-joining network analyses, in which isolates from Heilongjiang, Hebei and Xinjiang formed their own 
Table 3 Results of linkage disequilibrium analysis of allelic profile data from Cryptosporidium parvum at eight genetic loci

\begin{tabular}{|c|c|c|c|c|c|c|c|}
\hline Populations & $n$ & $P_{A}^{S}$ & $P_{M C}$ & $V_{D}$ & L & $V_{D}>L$ & LD or LE \\
\hline Guangdong & 10 & -0.0137 & 0.537 & 0.7091 & 1.3455 & No & LE \\
\hline Hebei & 12 & -0.0110 & 1 & 0.2564 & 0.5641 & No & LE \\
\hline Heilongjiang & 5 & - & 1 & - & - & - & - \\
\hline Jiangsu & 4 & 0.0286 & 1 & 0.2667 & 0.2667 & No & LE \\
\hline Shanghai & 10 & 0.0816 & 0.067 & 1.9727 & 2.0182 & No & LE \\
\hline Xinjiang & 5 & -0.0128 & 0.472 & 1.2111 & 3.2111 & No & LE \\
\hline IdA19G1 & 24 & 0.0272 & 0.175 & 1.2794 & 1.483 & No & LE \\
\hline IIA20G1 & 17 & 0.0867 & $<0.001$ & 0.9638 & 0.8008 & Yes & LD \\
\hline IdA15G1 & 5 & -0.0128 & 0.472 & 1.2111 & 3.2111 & No & LE \\
\hline Total & 46 & 0.0672 & $<0.001$ & 2.202 & 1.8848 & Yes & LD \\
\hline Guangdong $^{a}$ & 6 & -0.051 & 0.898 & 0.6 & 1.7429 & No & LE \\
\hline Hebei $^{\mathrm{a}}$ & 2 & - & - & - & - & - & - \\
\hline Heilongjianga & 1 & - & - & - & - & - & - \\
\hline Jiangsu ${ }^{a}$ & 2 & - & $<0.001$ & - & - & - & - \\
\hline Shanghaia & 7 & -0.0158 & 0.589 & 0.9476 & 2.2476 & No & LE \\
\hline Xinjiang ${ }^{a}$ & 3 & 0.2321 & 0.029 & 2.3333 & 2.3333 & No & LE \\
\hline$\| d A 19 G 1^{a}$ & 12 & -0.0695 & 0.986 & 0.5979 & 1.8287 & No & LE \\
\hline$\| \mathrm{dA} 20 \mathrm{G} 1^{\mathrm{a}}$ & 2 & - & $<0.001$ & - & - & - & - \\
\hline$\| d A 15 G 1^{a}$ & 3 & 0.2321 & 0.029 & 2.3333 & 2.3333 & No & LE \\
\hline Total $^{\mathrm{a}}$ & 17 & -0.0421 & 0.889 & 1.1307 & 2.3307 & No & LE \\
\hline
\end{tabular}

a Considering isolates with the same MLG as one individual

Abbreviations: $n$ number of isolates; $I_{A}^{S}$, standardized index of association calculated using the program LIAN $3.5 ; P_{M C}$, significance for obtaining this value in 1000 simulations using the Monte Carlo method; $V_{D}$, variance of pairwise difference; $L, 95 \%$ critical value for $V_{D} ; V_{D}>L$, presence of linkage disequilibrium

clusters while those from Shanghai, Jiangsu and Guangdong clustered together (Figs. 4 and 5).

The results of $F_{\text {st }}$ analysis supported the occurrence of geographically associated subpopulations of C. parvum IId subtypes. By gp60 subtype, isolates of IIdA15G1, IIdA19G1 and IIdA20G1 were genetically segregated from each other with high statistical significance (Table 4). Within the IIdA20G1 subtype, there was a significant differentiation between isolates from Hebei and Heilongjiang $\left(x^{2}=15.0, d f=1, P<0.0001\right)$. In contrast, the differentiation among IIdA19G1 isolates from Guangdong, Shanghai and Jiangsu was low. Compared with IIdA20G1 isolates from Heilongjiang, there was also reduced differentiation of between IIdA20G1 isolates from Hebei and IIdA19G1 isolates from Jiangsu and Shanghai (Table 5).

\section{Discussion}

The population genetic analysis of eight polymorphic loci has unravelled a high genetic diversity among isolates of C. parvum IId subtypes from different geographical areas in China. Although they were identical at the gp60 locus, the IIdA19G1 isolates differed at most other genetic loci including $d z$-hrgp, msc6-5, msc6-7, mucin1 and rpgr. Similarly, IIdA20G1 isolates from Hebei and
Heilongjiang differed from each other at the hsp 70 locus, while IIdA15G1 isolates from Xinjiang differed from each other at the $d z$-hrgp, hsp 70, msc6-5 and msc6-7 loci.

Results of the $L D$ analysis indicate the presence of an epidemic genetic structure of C. parvum IId subtypes in the present study. This could be attributed to the high prevalence of $C$. parvum in calves as the result of concentrated animal feeding operations and limited number of IId subtypes in China [2]. Indeed, IIdA19G1 and IIdA15G1 are dominant subtypes in cattle in China [7, 32]. Previously, isolates of $C$. parvum IId subtypes from China, Egypt and Sweden were shown to have a clonal population structure with limited genetic recombination [25]. The discrepancy in the inference of population genetic structure between these two studies was largely due to whether the analysis has taken the over-representation of the same MLG in the study population into consideration. If this had taken into consideration, the previously reported clonal population of C. parvum IId subtypes could be in fact an epidemic population.

Significant geographical segregation was observed in the IIdA15G1 isolates from Xinjiang and the IIdA20G1 isolates from Heilongjiang based on phylogenetic, substructure, PCoA and $F_{\text {st }}$ analyses. Previous reports indicated that most IIa isolates of C. parvum form 


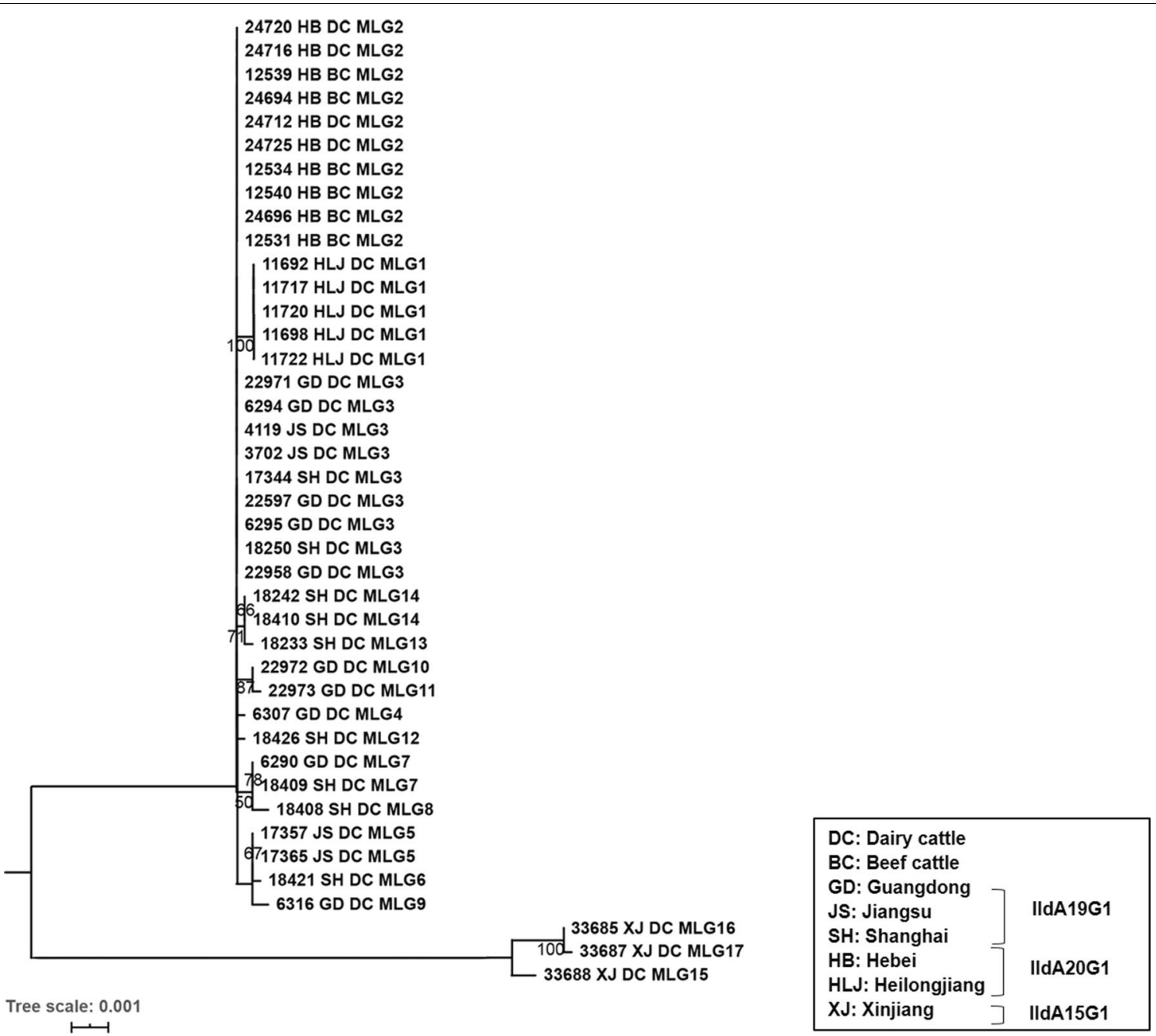

Fig. 2 Phylogenetic relationships among 41 Cryptosporidium parvum isolates. The phylogeny was inferred through maximum likelihood (ML) analysis of the concatenated nucleotide sequences based on substitution rates using the General Time Reversible model

country-specific populations. For example, an eBURSTbased analysis revealed geographical differences among isolates from Uganda, Israel, Serbia, Turkey and New Zealand [33]. Similarly, a significant geographical segregation was also identified among 692 C. parvum isolates from Italy, Ireland and Scotland [13]. The same situation was also observed in IId isolates of C. parvum between China and Sweden in a previous MLST study [25]. Other studies, however, have failed to identify geographical segregation in C. parvum populations, but they were conducted over smaller geographical areas within a country $[17,18]$. In the present study, diverse isolates were obtained from the southern, north-eastern and northwestern regions of China, leading to the identification of unique subpopulations of C. parvum in the more geographically isolated Xinjiang and Harbin. In contrast, isolates from Shanghai, Jiangsu and Guangdong had frequent genetic exchanges with no significant geographical barriers among them. These regions were chosen with the consideration of both geographical representation and the intensity of cattle production. The three C. parvum subtypes examined in the study are the most common ones in China, responsible for over $90 \%$ C. parvum infections in cattle. Additional population genetic analysis of more C. parvum isolates from other areas and other subtypes is needed to support the observations in this study. 

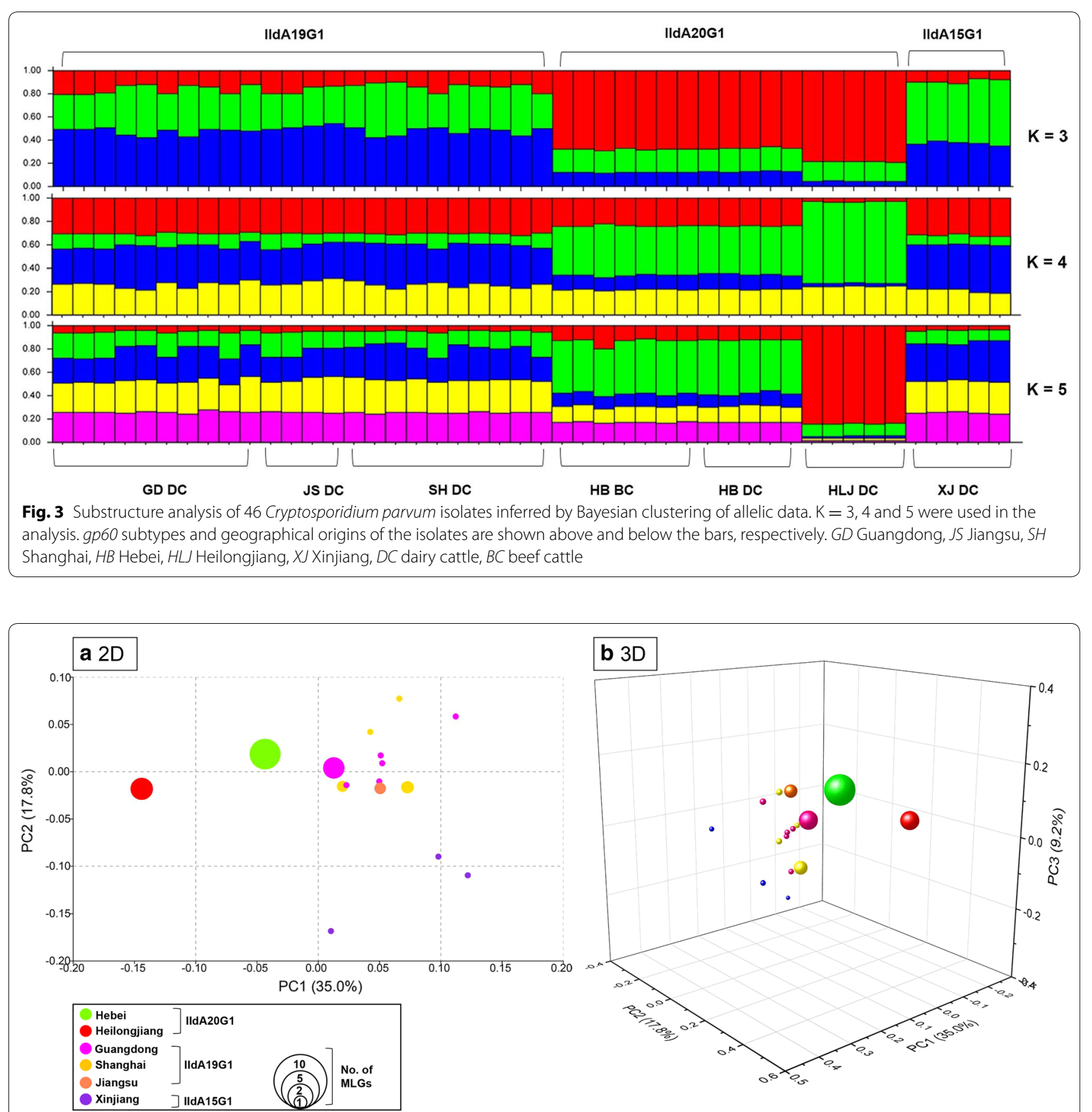

Fig. 4 Results of the principal coordinate analysis of 41 Cryptosporidium parvum isolates based on pairwise distances (a 2D, b 3D). Each solid sphere represents an MLG. The color of the spheres indicates geographical origin of the isolates, while the size of the spheres represents the number of isolates

The presence of multiple MLGs on almost all farms in Guangdong, Shanghai and Jiangsu suggests the presence of a significant intra-farm genetic diversity in C. par$v u m$. This was not revealed by gp60-based subtyping, as all isolates belonged to IIdA19G1. Nevertheless, this is in agreement with previous population genetic studies of C. parvum in European countries [12, 15, 19, 22, 25, 34]. This intra-farm genetic diversity of $C$. parvum in cattle 


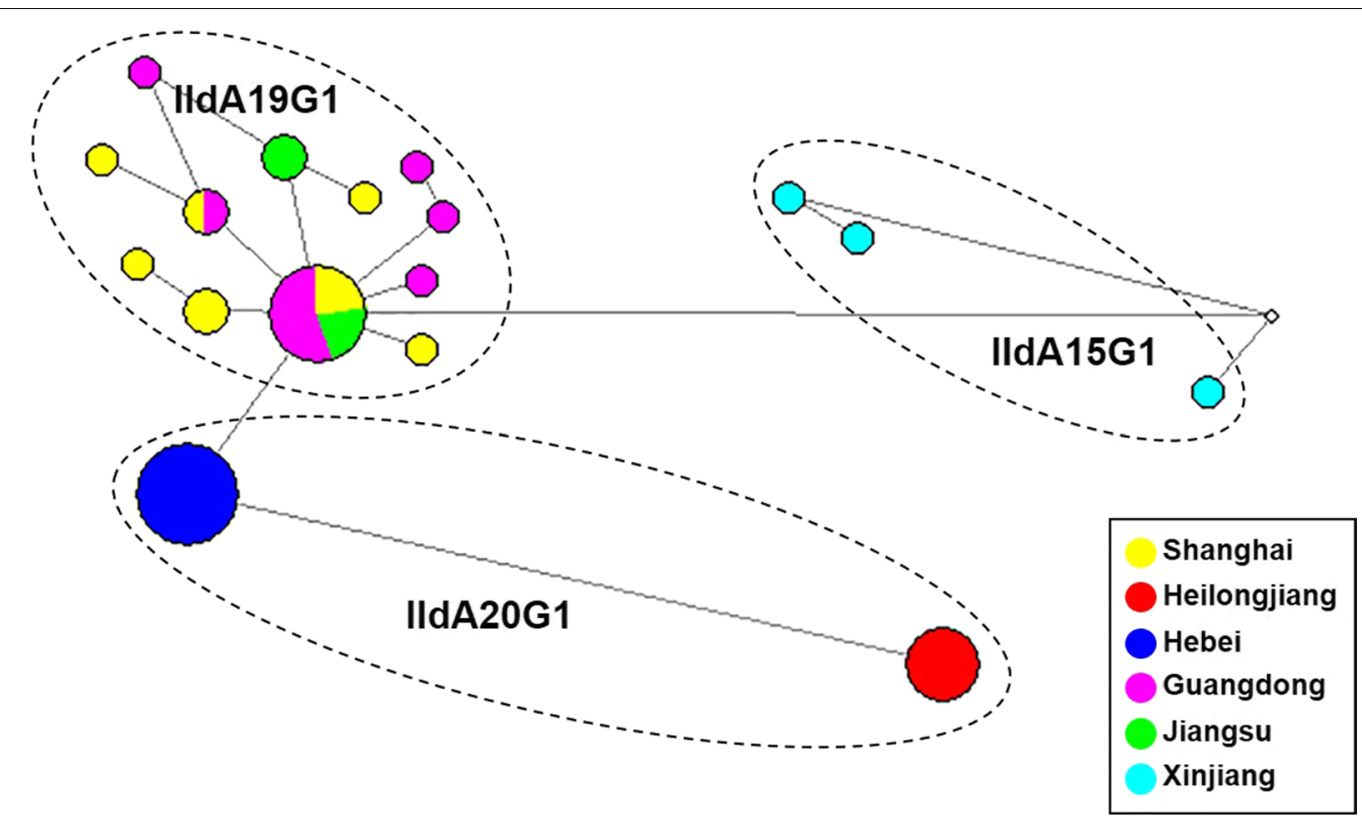

Fig. 5 Phylogeny of 41 Cryptosporidium parvum isolates inferred by median-joining network analysis. The size of each circle is proportional to the number of isolates with the MLG, while the color of the circles indicates the geographical origin of the MLG

Table 4 Pairwise genetic distance $\left(F_{\text {st }}\right.$, below the diagonal) and $P$-values based on Chi-square test (above the diagonal) between subpopulations of Cryptosporidium parvum by gp60 subtype

\begin{tabular}{llll}
\hline Subtype & IdA19G1 & IdA20G1 $\left(X^{2}, d f, P\right)$ & IdA15G1 $\left(X^{2}, d f, P\right)$ \\
\hline IdA19G1 & & $(18.2,12,<0.0001)$ & $(26.0,14,<0.0001)$ \\
IIA20G1 & 0.14262 & & $(18.0,4,<0.0001)$ \\
IdA15G1 & 0.34028 & 0.32851 & \\
\hline
\end{tabular}

may be attributed to frequent animal trade among farms, which is known to increase the heterogeneity of $C$. par$v u m$ and the complexity of infections $[14,19,21]$.

\section{Conclusions}

Despite the presence of only a limited number of gp60 subtypes of C. parvum in cattle in China, a much higher genetic diversity is evident in MLST characterization of isolates at both farm and region levels. Nevertheless, biological selection has led to the dominance of limited numbers of geographically segregated MLGs of C. parvum in calves in China, with an apparent epidemic population structure. Currently, the veterinary and public health significance of this biological selection of C. parvum subpopulations is not entirely clear. Efforts should be made to monitor the genetic evolution of this unique zoonotic pathogen in China.

Table 5 Pairwise genetic distance $\left(F_{\mathrm{st}}\right.$, below the diagonal) and $P$-values based on Chi-square test (above the diagonal) between subpopulations of Cryptosporidium parvum by geographical origin

\begin{tabular}{|c|c|c|c|c|c|c|}
\hline Location & Guangdong & Jiangsu $\left(x^{2}, d f, P\right)$ & Shanghai $\left(X^{2}, d f, P\right)$ & Hebei $\left(X^{2}, d f, P\right)$ & Heilongjiang $\left(X^{2}, d f, P\right)$ & Xinjiang $\left(X^{2}, d f, P\right)$ \\
\hline Guangdong & & $\begin{array}{l}(8.3,7,0.00901 \pm \\
0.0091)\end{array}$ & $\begin{array}{l}(11.3,10,0.02703 \pm \\
\quad 0.0194)\end{array}$ & $(6.7,5,0.23423 \pm 0.0411)$ & $(3.8,5,<0.0001)$ & $(13.0,8,<0.0001)$ \\
\hline Jiangsu & 0.14401 & & $(8.3,7,0.04505 \pm 0.0244)$ & $(5.8,1,0.01802 \pm 0.0121)$ & $(3.2,1,<0.0001)$ & $(7.0,4,<0.0001)$ \\
\hline Shanghai & 0.05817 & 0.09422 & & $\begin{array}{l}(12.3,6,0.01802 \pm \\
\quad 0.0121)\end{array}$ & $(7.8,6,<0.0001)$ & $(12.0,9,<0.0001)$ \\
\hline Hebei & 0.02455 & 0.32678 & 0.08043 & & $(15.0,1,<0.0001)$ & $(13.0,3,<0.0001)$ \\
\hline Heilongjiang & 0.67935 & 0.85765 & 0.5247 & 0.87905 & & $(8.0,3,<0.0001)$ \\
\hline Xinjiang & 0.36149 & 0.29526 & 0.28499 & 0.36733 & 0.47694 & \\
\hline
\end{tabular}




\section{Supplementary information}

Supplementary information accompanies this paper at https://doi. org/10.1186/s13071-020-04303-y.

Additional file 1: Table S1. Cryptosporidium parvum isolates used in this study and their genetic identity at eight loci.

\section{Abbreviations}

PCR: Polymerase chain reaction; gp60: 60-kDa glycoprotein; msc6-7: Serine repeat antigen; rpgr: Retinitis pigmentosa GTPase regulator; msc6-5: Hypothetical trans-membrane protein; dz-hrgp: Hydroxyproline-rich glycoprotein chom3t: T-rich gene fragment; hsp70: 70-kDa heat-shock protein; mucin 1: Mucin-like protein; LD: Linkage disequilibrium; LE: Linkage equilibrium; MLG: Multi-locus genotype; Rms: Recombination events.

\section{Acknowledgements}

We thank the farm owners and staff for their assistance in sample collection during this study.

\section{Authors' contributions}

$Y F$ and $L X$ conceived and designed the experiments. ZZ performed the experiments. SH, WZ, YG, NL, ZZ, LZ and MK provided technical assistance. ZZ, SH, YF and LX analyzed the data. ZZ, YF and LX wrote the paper. All authors read and approved the final manuscript.

\section{Funding}

This study was supported by the National Natural Science Foundation of China (U1901208, 31820103014) and the 111 Project (D20008).

\section{Availability of data and materials}

The data supporting the conclusions of this article are included within the article. Representative nucleotide sequences generated from this study were deposited in the GenBank database under the accession numbers MT303107-MT303131.

\section{Ethics approval and consent to participate}

This study was performed strictly according to the recommendations of the Guide for the Care and Use of Laboratory Animals of the Ministry of Health, China. Animals sampled were handled following established procedures of the Chinese Laboratory Animal Administration Act of 2017. The research protocol was reviewed and approved by the Research Ethics Committee of the South China Agricultural University.

\section{Consent for publication}

Not applicable.

\section{Competing interests}

The authors declare that they have no competing interests.

\section{Author details}

${ }^{1}$ Center for Emerging and Zoonotic Diseases, College of Veterinary Medicine South China Agricultural University, Guangzhou, Guangdong 510642, China.

${ }^{2}$ Guangdong Laboratory for Lingnan Modern Agriculture, Guangzhou 510642, China. ${ }^{3}$ College of Animal Science and Veterinary Medicine, Henan Agricultural University, Zhengzhou 450002, China. ${ }^{4}$ Institute of Parasitology, Biology Centre of the Academy of Sciences of the Czech Republic, České Budějovice 37005, Czech Republic.

Received: 19 April 2020 Accepted: 10 Auqust 2020

Published online: 18 August 2020

\section{References}

1. Checkley W, White AC, Jaganath D, Arrowood MJ, Chalmers RM, Chen $X$, Fayer $R$, et al. A review of the global burden, novel diagnostics, therapeutics, and vaccine targets for Cryptosporidium. Lancet Infect Dis. 2015:15:85-94.
2. Feng Y, Ryan UM, Xiao L. Genetic diversity and population structure of Cryptosporidium. Trends Parasitol. 2018;34:997-1011.

3. Xiao L, Feng Y. Molecular epidemiologic tools for waterborne pathogens Cryptosporidium sp and Giardia duodenalis. Food Waterborne Parasitol. 2017:8-9:14-32.

4. Nader JL, Mathers TC, Ward BJ, Pachebat JA, Swain MT, Robinson G, et al. Evolutionary genomics of anthroponosis in Cryptosporidium. Nat Microbiol. 2019;4:826-36.

5. Ryan U, Fayer R, Xiao L. Cryptosporidium species in humans and animals: current understanding and research needs. Parasitology. 2014;141:1667-85.

6. Xiao L. Molecular epidemiology of cryptosporidiosis: an update. Exp Parasitol. 2010;124:80-9.

7. Feng Y, Xiao L. Molecular epidemiology of cryptosporidiosis in China. Front Microbiol. 2017;8:1701-11.

8. Li N, Wang R, Cai M, Jiang W, Feng Y, Xiao L. Outbreak of cryptosporidiosis due to Cryptosporidium parvum subtype IIAA19G1 in neonatal calves on a dairy farm in China. Int J Parasitol. 2019;49:569-77.

9. Cui Z, Wang R, Huang J, Wang H, Zhao J, Luo N, et al. Cryptosporidiosis caused by Cryptosporidium parvum subtype IIdA15G1 at a dairy farm in northwestern China. Parasit Vectors. 2014;7:529.

10. Widmer G, Lee Y. Comparison of single- and multilocus genetic diversity in the protozoan parasites Cryptosporidium parvum and C. hominis. Appl Environ Microb. 2010;76:6639-44.

11. Chalmers RM, PérezCordón G, Cacció SM, Klotz C, Robertson LJ. Participants of the Cryptosporidium genotyping workshop (EURO-FBP) Cryptosporidium genotyping in Europe: the current status and processes for a harmonised multi-locus genotyping scheme. Exp Parasitol. 2018;191:25-30.

12. Mallon ME, MacLeod A, Wastling JM, Smith H, Tait A. Multilocus genotyping of Cryptosporidium parvum Type 2: population genetics and substructuring. Infect Genet Evol. 2003;3:207-18.

13. Cacció SM, de Waele V, Widmer G. Geographical segregation of Cryptosporidium parvum multilocus genotypes in Europe. Infect Genet Evol. 2015;31:245-9.

14. De Waele V, Van den Broeck F, Huyse T, McGrath G, Higgins I, Speybroeck $\mathrm{N}$, et al. Panmictic structure of the Cryptosporidium parvum population in Irish calves: influence of prevalence and host movement. Appl Environ Microbiol. 2013;79:2534-41.

15. Drumo R, Widmer G, Morrison LJ, Tait A, Grelloni V, D'Avino N, et al. Evidence of host-associated populations of Cryptosporidium parvum in Italy. Appl Environ Microbiol. 2012;78:3523-9.

16. Feng Y, Torres E, Li N, Wang L, Bowman D, Xiao L. Population genetic characterisation of dominant Cryptosporidium parvum subtype llaA15G2R1. Int J Parasitol. 2013:43:1141-7.

17. Herges GR, Widmer G, Clark ME, Khan E, Giddings CW, Brewer M, et al. Evidence that Cryptosporidium parvum populations are panmictic and unstructured in the Upper Midwest of the United States. Appl Environ Microbiol. 2012;78:8096-101.

18. Mallon M, MacLeod A, Wastling J, Smith H, Reilly B, Tait A. Population structures and the role of genetic exchange in the zoonotic pathogen Cryptosporidium parvum. J Mol Evol. 2003;56:407-17.

19. Morrison LJ, Mallon ME, Smith HV, MacLeod A, Xiao L, Tait A. The population structure of the Cryptosporidium parvum population in Scotland: a complex picture. Infect Genet Evol. 2008;8:121-9.

20. Ramo A, Quílez J, Monteagudo L, Del Cacho E, Sánchez-Acedo C. Intraspecies diversity and panmictic structure of Cryptosporidium parvum populations in cattle farms in northern Spain. PloS ONE. 2016;11:e0148811.

21. Tanriverdi S, Markovics A, Arslan MO, Itik A, Shkap V, Widmer G. Emergence of distinct genotypes of Cryptosporidium parvum in structured host populations. Appl Environ Microbiol. 2006;72:2507-13.

22. Ramo A, Monteagudo LV, Del Cacho E, Sánchez-Acedo C, Quílez J. Intraspecies genetic diversity and clonal structure of Cryptosporidium parvum in sheep farms in a confined geographical area in northeastern Spain. PloS One. 2016;11:e0155336.

23. Feng Y, Gong X, Zhu K, Li N, Yu Z, Guo Y, et al. Prevalence and genotypic identification of Cryptosporidium spp., Giardia duodenalis and Enterocytozoon bieneusi in pre-weaned dairy calves in Guangdong, China. Parasit Vectors. 2019;12:41. 
24. Cai M, Guo Y, Pan B, Li N, Wang X, Tang C, et al. Longitudinal monitoring of Cryptosporidium species in pre-weaned dairy calves on five farms in Shanghai China. Vet Parasitol. 2017;241:14-9.

25. Wang R, Zhang L, Axen C, Bjorkman C, Jian F, Amer S, et al. Cryptosporidium parvum Ild family: clonal population and dispersal from western Asia to other geographical regions. Sci Rep. 2014;4:4208.

26. Rozas J, Ferrer-Mata A, Sanchez-DelBarrio JC, Guirao-Rico S, Librado P, Ramos-Onsins SE, et al. DnaSP 6: DNA sequence polymorphism analysis of large data sets. Mol Biol Evol. 2017:34:3299-302.

27. Haubold B, Hudson RR. LIAN 3.0: detecting linkage disequilibrium in multilocus data. Linkage analysis. Bioinformatics. 2000;16:847-8.

28. Stamatakis A. RAxML version 8: a tool for phylogenetic analysis and postanalysis of large phylogenies. Bioinformatics. 2014;30:1312-3.

29. Hubisz MJ, Falush D, Stephens M, Pritchard JK. Inferring weak population structure with the assistance of sample group information. Mol Ecol Resour. 2009;9:1322-32.

30. Peakall R, Smouse PE. GenAIEx 6.5: genetic analysis in Excel. Population genetic software for teaching and research —an update. Bioinformatics. 2012;28:2537-9.
31. Bandelt HJ, Forster P, Rohl A. Median-joining networks for inferring intraspecific phylogenies. Mol Biol Evol. 1999;16:37-48.

32. Wang R, Zhao G, Gong Y, Zhang L. Advances and perspectives on the epidemiology of bovine Cryptosporidium in China in the past 30 years. Front Microbiol. 2017:8:1823.

33. Tanriverdi S, Grinberg A, Chalmers RM, Hunter PR, Petrovic Z, Akiyoshi DE, et al. Inferences about the global population structures of Cryptosporidium parvum and Cryptosporidium hominis. Appl Environ Microbiol. 2008;74:7227-34.

34. Quílez J, Vergara-Castiblanco C, Monteagudo L, Del Cacho E, SánchezAcedo C. Multilocus fragment typing and genetic structure of Cryptosporidium parvum isolates from diarrheic preweaned calves in Spain. Appl Environ Microbiol. 2011;77:7779-86.

\section{Publisher's Note}

Springer Nature remains neutral with regard to jurisdictional claims in published maps and institutional affiliations.
Ready to submit your research? Choose BMC and benefit from:

- fast, convenient online submission

- thorough peer review by experienced researchers in your field

- rapid publication on acceptance

- support for research data, including large and complex data types

- gold Open Access which fosters wider collaboration and increased citations

- maximum visibility for your research: over $100 \mathrm{M}$ website views per year

At BMC, research is always in progress.

Learn more biomedcentral.com/submissions 